\title{
Percepción sobre los libros de texto en la enseñanza-aprendizaje de la Matemática
}

\section{Perception about textbooks in math teaching-learning of Mathematics}

\author{
Ángel Montaluisa-Vivas \\ Universidad Central del Ecuador, Quito, Ecuador \\ aemontaluisa@uce.edu.ec \\ https://orcid.org/0000-0002-6037-2127
}

Edgar Salas-Jaramillo

Universidad Central del Ecuador, Quito, Ecuador esalasj2003@yahoo.com.ar https://orcid.org/0000-0003-3391-4979

Leonardo Canga-Unda

Universidad Central del Ecuador, Quito, Ecuador lncanga@uce.edu.ec https://orcid.org/0000-0002-9576-7583

Adriana Ponce-Benavides

Universidad Central del Ecuador, Quito, Ecuador acponceb@uce.edu.ec https://orcid.org/0000-0003-1419-7636

(Recibido: 01/03/2021; Aceptado: 15/03/2021; Versión final recibida: 15/04/2021)

Cita del artículo: Montaluisa-Vivas, A., Salas-Jaramillo, E., Canga-Unda L. y Ponce-Benavides, A. (2021). Percepción sobre los libros de texto en la enseñanza-aprendizaje de la Matemática. Revista Cátedra, 4(2), 55-69.

\section{Resumen}

Los libros de texto son un recurso necesario en el proceso de enseñanza-aprendizaje de las ciencias y deben considerar, entre otros, a los elementos curriculares: objetivos de

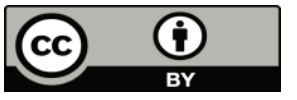


aprendizaje, contenidos matemáticos y evaluación. Esta investigación tiene como propósito determinar el nivel de satisfacción que el desarrollo de estos elementos genera en estudiantes y docentes, expresados en estos libros que se utilizan en el sistema educativo ecuatoriano. Cabe recalcar que el Ministerio de Educación dota de manera gratuita este material escolar a todas las instituciones públicas del país y, por lo tanto, motiva a investigar sobre su aplicación y uso. Este estudio tiene enfoque cuantitativo y es de nivel exploratorio y descriptivo, por lo que se usó un instrumento de observación para la recolección de datos. Los resultados se expresan por niveles de escolaridad tanto de la Educación General Básica subnivel Superior como del Bachillerato General Unificado y por los elementos curriculares estudiados. Finalmente, se concluyó que los objetivos propuestos para el Bachillerato General Unificado tienen un nivel de satisfacción mayor que los declarados para la Educación General Básica, asimismo, se concluyó para ambos niveles que existe un mediano nivel de satisfacción de los elementos investigados en los libros de texto, lo que significa que los estudiantes que los utilizan no están orientados para continuar aprendizajes más complejos.

\section{Palabras clave}

Currículo, enseñanza-aprendizaje, evaluación, Matemática, objetivos de aprendizaje, textos escolares.

\section{Abstract}

Textbooks are a necessary resource in the science teaching-learning process and should consider, among others, the curricular elements: learning objectives, mathematical content and evaluation. The purpose of this research is to determine the level of satisfaction that the development of these elements generates in students and teachers, expressed in these textbooks used in the Ecuadorian educational system. It should be noted that the Ministry of Education provides this school material free of charge to all public institutions in the country and, therefore, motivates research on its application and use. This study has a quantitative approach and is exploratory and descriptive, so an observation instrument was used for data collection. The results are expressed by levels of schooling of both the General Basic Education sub-level and the General Unified Baccalaureate and by the curricular elements studied. Finally, it was concluded that the objectives proposed for the Unified General Baccalaureate have a higher level of satisfaction than those declared for General Basic Education. It was also concluded for both levels that there is a medium level of satisfaction with the elements investigated in the textbooks, which means that the students who use them are not oriented to continue with more complex learning.

\section{Keywords}

Curriculum, evaluation, learning objectives, Math, school texts, teaching-learning.

\section{Introducción}

La evaluación aplicada a los estudiantes del país señala que en la asignatura de Matemática no alcanzan los niveles mínimos de aprobación, con relación a los estándares propuestos por el Ministerio de Educación (MINEDUC). Como afirma Puente, los resultados de las pruebas Ser Bachiller en el período 2015-2016, aplicadas a más de cien mil estudiantes, fueron: Matemáticas 743 puntos, Lengua y Literatura 786 puntos, Ciencias Naturales 771 puntos y Estudios Sociales 856 puntos (Puente, 2016, p. 1). Entonces, ante tales resultados cabe preguntarse: ¿qué factores inciden en estos bajos puntajes? Las respuestas son múltiples y de diversa índole: sociales, culturales, económicas, escolares, familiares, 
personales, entre otras. Además, están directamente relacionadas con aspectos del proceso de enseñanza-aprendizaje: la limitada formación y capacitación de los docentes en Matemática y didáctica de la Matemática, los recursos con los que se cuentan no aportan en el desarrollo de los contenidos de la asignatura, los estudiantes tienen temores y desmotivación, los procesos educativos de aula están mal concebidos y aplicados, los instrumentos de evaluación no son los pertinentes, entre muchos otros.

El proceso de enseñanza-aprendizaje demanda el uso de recursos didácticos, de los muchos que existen. A pesar de la variedad de recursos y de los avances tecnológicos ligados al ámbito educativo, especialmente en el área de Matemática, los libros de texto continúan siendo un apoyo indispensable durante el proceso. "El libro de texto es un recurso importante para llevar a cabo el curriculum y la programación didáctica de las matemáticas con eficacia, pero no es el único" (García, 2014, p. 1). Los docentes emplean los libros de texto para desarrollar los contenidos de la asignatura que están organizados en las mallas curriculares de cada año lectivo, así como los estudiantes disponen de los textos entregados gratuitamente y deben utilizarse de manera obligatoria en el desarrollo de las clases de Matemática.

En cuanto a la estructura, los libros de texto entregados a todos los estudiantes de los niveles de Educación General Básica (EGB) Superior y Bachillerato General Unificado (BGU) presentan las siguientes características: ediciones del año 2016, su autoría es responsabilidad del MINEDUC, están organizados en unidades temáticas y tienen un número promedio de páginas de 283 en el EGB Superior y 255 en el BGU. Finalmente, consideran la portada, índice, contenidos, glosario, bibliografía y webgrafía. La revisión de los libros de texto fue realizada por la Universidad Politécnica Salesiana. La sinopsis de todos los libros de texto expresa lo siguiente:

Este libro de texto que tienes en tus manos es una herramienta muy importante para que puedas desarrollar los aprendizajes de la mejor manera. Un libro de texto no debe ser la única fuente de investigación y de descubrimiento, pero siempre es un buen aliado que te permite descubrir por ti mismo la maravilla de aprender. El Ministerio de Educación ha realizado un ajuste curricular que busca mejores oportunidades de aprendizaje para todos los estudiantes del país en el marco de un proyecto que propicia su desarrollo personal pleno y su integración en una sociedad guiada por los principios del Buen Vivir, la participación democrática y la convivencia armónica (Ministerio de Educación del Ecuador, 2016, p. 1).

Bajo estos planteamientos, se formulan las siguientes preguntas de investigación: ¿de qué manera los libros de texto utilizados por los estudiantes del EGB subnivel Superior y del BGU están estructurados?, y ¿los libros de texto para el aprendizaje de Matemática satisfacen y son coherentes entre los objetivos, los contenidos y la evaluación? Para dar respuesta a estas interrogantes, el objetivo general de la investigación es determinar el nivel de satisfacción que genera el desarrollo de objetivos, contenidos y evaluación expresados en los libros de texto utilizados en la EGB Superior y en el BGU del sistema educativo ecuatoriano. A partir de él, se desglosan los siguientes objetivos específicos:

1. Identificar los elementos curriculares: objetivos, contenidos y evaluación, aplicados en los libros de texto tanto del nivel EGB Superior como del BGU.

2. Establecer de qué manera se desarrollan los contenidos matemáticos en los libros de texto. 
3. Describir el nivel de satisfacción que se establecen, por años, en los elementos curriculares formulados en los libros de texto.

Pese a las reformas educativas de los últimos años, Fernández y Caballero enfatizan que la utilización de los libros de texto de Matemática es y seguirá siendo una práctica generalizada en las aulas de clase. Es imperativo su fundamentación en las experiencias del estudiante y su vinculación con los avances e innovaciones en el campo científico (Fernández y Caballero, 2017, p. 202). Por lo tanto, analizar los objetivos de aprendizaje, contenidos y evaluación de este recurso permite determinar si cumple con las expectativas, de forma que los estudiantes tengan un respaldo metodológico para alcanzar conocimientos y desarrollar razonamientos y aprendizajes matemáticos en relación con el nivel de escolaridad y su respectiva secuencialidad.

De acuerdo con Fernández y Caballero, un libro de texto no sustituye al docente, no limita el desarrollo de metodologías y no convierte al aprendizaje en un proceso de repetición y memorismo (Fernández y Caballero, 2017, p. 205). Al contrario, diversificar el uso de recursos didácticos en el proceso de enseñanza-aprendizaje de Matemática aporta al cumplimiento de los logros esperados y a hacer frente a las demandas actuales de la educación del siglo XXI. Bajo esta perspectiva, la investigación acerca de los libros de texto de Matemática exige profundizar en los objetivos, contenidos y evaluación de los aprendizajes, abordar el estudio desde diferentes disciplinas y contextos, generar espacios de discusión y orientar este recurso hacia un enfoque interdisciplinar.

La presente investigación se desarrolla con la siguiente estructura: revisión de la literatura, donde se fundamenta acerca de los libros de texto de Matemática, especificando sobre sus objetivos de aprendizaje, contenidos matemáticos y evaluación; métodos y materiales, en el que se detalla sobre el diseño del estudio y su instrumento de recolección de datos; resultados y discusión, agrupados por los elementos curriculares nombrados como criterios (objetivos, contenidos y evaluación) y niveles de escolaridad (EGB Superior y BGU); y, por último, se establecen las conclusiones pertinentes.

\section{Revisión de la literatura}

En la Constitución de la República del Ecuador se expresa la gratuidad de la educación pública y la obligatoriedad para todos los niños, niñas y adolescentes de asistir y cumplir con la Educación General Básica y el Bachillerato (Constitución de la República del Ecuador, 2008, Artículo 28). Con esta decisión, entre algunas medidas, se entregan gratuitamente los libros de texto para las áreas fundamentales: Matemática, Lengua y Literatura, Ciencias Sociales y Ciencias Naturales, por lo que los libros de texto se han generalizado en estos niveles. Además, estos textos cumplen diferentes papeles: objeto de estudio, material de consulta, registro de las actividades del alumno, colección de ejercicios propuestos y problemas a resolver. Choppin (1993) considera que el libro de texto es:

A la vez apoyo del saber en tanto que impone una distribución y una
jerarquía de los conocimientos y contribuye a forjar los andamios
intelectuales tanto de alumnos como de profesores; es instrumento de
poder, dado que contribuye a la uniformización lingüística de la
disciplina, a la nivelación cultural y a la propagación de las ideas domi-
nantes (pp. 165-185).

Los libros de texto entregados para la enseñanza de la Matemática también deben cumplir con las orientaciones que se expresan en el Nuevo Currículo 2016 para la EGB y el BGU. Los libros de texto, para Nortes y Nortes (2011), "están encaminados a desarrollar los 
contenidos establecidos por los decretos educativos correspondientes a los distintos niveles de la enseñanza obligatoria [y concluyen que] han desempeñado un papel preponderante en el proceso de enseñanza-aprendizaje de la matemática" (pp. 68-69). De igual manera, García (2014) expresa que "el libro de texto de matemáticas era y todavía es considerado como uno de los recursos más importantes para la enseñanza y el aprendizaje de las matemáticas" (p. 4).

También, cabe tomar en cuenta que la Unesco, a través de Flotts et al. realizó un estudio que comparó y analizó los documentos curriculares de Matemática de los países participantes en el Tercer Estudio Regional Comparativo y Explicativo (TERCE) y otros insumos, como libros de texto (Flotts et al., 2016, p. 26). También, permite reconocer el desarrollo de la capacidad de resolver problemas como eje central de la formación matemática.

Según Standaert y Troch (2011), "un buen uso del libro de texto, además, depende, en buena medida, de la actitud del docente y de la situación de partida de los estudiantes" (p. 108), por lo que un adecuado proceso de clase se iniciará con la revisión de los prerrequisitos y de la motivación de los participantes. Los libros de textos cobran importancia cuando ellos, por sí mismos, cautivan a los estudiantes a utilizarlos.

En lo referente a las características de un buen libro de texto, Creemers menciona las siguientes:

1. Aporta ejercicios prácticos que responden al objetivo de la temática de estudio.

2. La materia de estudio no aparece sobrecargada.

3. Los contenidos responden a los objetivos de enseñanza y son aplicables en situaciones de la vida real.

4. Los objetivos aparecen claramente expresados, están organizados e informan a los estudiantes sobre lo que se espera que aprendan.

5. La materia de estudio se explica de forma clara y estructurada y responde a los objetivos. Además, se presenta en una secuencia lógica, que va desde lo más simple hasta lo más complejo.

6. Utilizan advance organizers (noción introducida por Ausubel) y se trata de un elemento explicativo que aparece al inicio de cada nuevo capítulo y que conecta las nociones ya estudiadas con las que aparecen a continuación.

7. Aportan material para el seguimiento y la evaluación del progreso del estudiante.

8. Se demanda la retroalimentación de manera sistemática.

9. Permiten el uso efectivo del tiempo de clase.

10. Promueven la motivación de los estudiantes.

11. Aportan material para la evaluación formativa (Creemers, 1991, p. 48).

\subsection{Los objetivos de aprendizaje}

Los objetivos propuestos en los libros de texto son propósitos que se formulan para determinar lo que se hará o tratará de hacer en clase, en otras palabras, son "aspiraciones o intenciones que pueden ser muy interesantes y que seguramente expresen la necesidad de que el alumno adquiera determinados conocimientos, habilidades, actitudes y/o destrezas" (Salcedo, 2011, p. 117). Los procesos de aprendizaje que se aplican exigen el uso de libros de texto que apoyen la consecución de los objetivos de aprendizaje; por ello, Van Bruggen (1987) afirma que, "cuanto más claros son los objetivos centrales y los contenidos, más fácil será medir el impacto de los libros de texto en los logros del aprendizaje" (p. 133).

Por consiguiente, la Matemática escolar se enfoca en privilegiar su aspecto formativo. Así también, "los objetivos comprenden las experiencias que los educandos deben ganar en todas las oportunidades de aprendizaje ofrecidas. Estos influyen en la selección de 
contenidos, estrategias metodológicas y recursos, y en la evaluación de los aprendizajes" (Araujo, 2009, p. 9). Con ello, se debe considerar que los libros de texto son recursos de apoyo que sirven para alcanzar los logros que los estudiantes deben evidenciar al término de una clase.

En el informe de la Unesco, a través de Flotts et al., se expresa que un objetivo de la enseñanza de la Matemática es formar personas capaces de razonar lógicamente y de pensar críticamente, que dominan ciertos saberes o contenidos propios de esta disciplina y sean capaces de aplicarlos en la vida cotidiana (Flotts et al., 2016, p. 26). En la etapa educativa de EGB subnivel Superior y BGU, los adolescentes deben apreciar y entender la significatividad de los números y usarlos con propiedad, así como las distintas finalidades que tienen en la vida cotidiana. La importancia numérica se deriva de experiencias y acciones sobre situaciones reales, de modo que, progresivamente, los estudiantes emplean números para cuantificar, medir, indicar, ordenar, ubicar, entre otros; y desarrollan operaciones numéricas para resolver problemas de esta naturaleza.

\subsection{Los contenidos matemáticos}

Los libros de texto en Ecuador son recursos didácticos de uso casi obligatorio por parte de los principales actores involucrados en el proceso de enseñanza-aprendizaje. Parcerisa sostiene que los libros de texto condicionan el tipo de enseñanza que se realiza cuando son utilizados de manera cerrada y sometiendo a los estudiantes a los contenidos de aprendizaje y metodologías del Currículo Nacional prestablecidas en el documento (Parcerisa, 1996, p. 35). Velásquez (2019) según estudios realizados sobre textos escolares, añade lo siguiente:

Los estudiantes, maestros, padres y representantes, les otorgan una posición de alta estima. No obstante, esa dependencia "libresca" es preocupante, porque se asume la veracidad casi total de los contenidos y se presume, con pasividad, de las buenas intenciones pedagógicodidácticas de sus mentores (p. 19).

Como contraparte, se tiene que, para Eyzaguirre y Fontaine (1997), "la metodología y los contenidos de los textos de estudio pueden llegar a jugar un papel muy relevante si se quiere una reforma educacional razonable, realista, rápida, de amplio alcance y compatible con una sociedad pluralista" (p. 340). De este modo, los libros de texto para el aprendizaje de la Matemática deben incorporar contenidos de la asignatura que guarden una secuencia lógica, tanto en lo teórico como en lo didáctico.

Por ello, hay que establecer relaciones y conexiones entre elementos, acciones, nociones y conceptos, lo que permitirá saber las condiciones que se deben cumplir para desarrollar la capacidad de secuenciar y comprender los hechos. En el proceso de enseñanza-aprendizaje, Obaya y Ponce señalan que la secuencia didáctica orienta y facilita el desarrollo práctico. También, fomenta la investigación como herramienta metodológica que permite la construcción de conceptos, procedimientos y actitudes con carácter de una propuesta flexible que puede y debe adaptarse a la realidad concreta (Obaya y Ponce, 2007, p. 19).

\subsection{La evaluación}

Se define a la evaluación como "una fase de control que tiene como objeto no sólo la revisión de lo realizado sino también el análisis sobre las causas y razones para determinados resultados" (Duque, 1993, p. 167). Además, "la evaluación adquiere sentido cuando es capaz de generar información que sirva para tomar decisiones e iluminar las acciones de mejora" (Flotts et al., 2016, p. 9). En el ámbito educativo coexisten la evaluación formativa y la evaluación sumativa o certificadora, las cuales se ligan al aprendizaje que se imparte en el aula. Arribas (2017) define lo siguiente: 
La evaluación en su dimensión pedagógica, formativa, como un elemento más del proceso de enseñanza aprendizaje se manifiesta más netamente cuando la evaluación no lleva aparejada una calificación o cuando esta no tiene repercusiones más allá de la valoración del progreso del interesado (p. 383).

En consecuencia, la evaluación formativa se caracteriza por ser orientadora, reguladora y motivadora; se inclina a valorar la formación del estudiante y su manera de aprender. Se realiza un seguimiento de su trayectoria, desde su punto de partida hasta su punto de llagada, para mejorar el proceso educativo con respecto a los datos adquiridos; no requiere de una calificación para valorarse.

Para Arribas, la evaluación en su dimensión sumativa o certificadora requiere constatar el grado de consecución de los objetivos de aprendizaje por parte del estudiante, con el fin de avalar, clasificar, revisar, retomar y reflexionar en función de los resultados obtenidos (Arribas, 2017, p. 384). Al contrario de la evaluación formativa, la evaluación sumativa necesariamente cuenta con una calificación para determinar el nivel de logro obtenido en cada proceso de aprendizaje.

La correspondencia y complementariedad entre evaluación formativa y sumativa otorgan a que el proceso evaluativo sea sistemático, participativo, continuo e interpretativo, en lo cualitativo y cuantitativo. En relación a ello, Villardón (2006) considera que:

La evaluación en su función sumativa como evaluación de competencias y la evaluación formativa como evaluación para el desarrollo de competencias [son] dos enfoques complementarios y necesarios de la evaluación de los aprendizajes, que conducen a una concepción global de lo que debe ser la evaluación [...] como elemento de la formación competencial (pp. 61-62).

Un libro de texto, para Campanario, es una fuente de información, tareas, preguntas y ejercicios de evaluación, para ser revisados por docentes y estudiantes dentro y fuera de clases (Campanario, 2001, p. 352). De manera análoga, García (2014) sostiene que "los principales usos que los profesores dan a los libros discurren entre fuente de información para preparar, impartir y evaluar las clases y como base de datos con tareas y ejercicios que los alumnos han de resolver" (p. 10). En los libros de texto de Matemática, la evaluación de los aprendizajes toma mayor fuerza en las actividades propuestas que desarrollan los estudiantes.

Específicamente, el planteamiento y enunciados de los problemas que se encuentran en los libros de texto de Matemática influyen en la percepción y entendimiento del estudiante para resolverlo, así como del docente para explicarlo. Fernández (2010) pone en relieve las consecuencias de tener problemas enunciados de forma incorrecta en el quehacer educativo en el aula de Matemática.

Cuando se presenta en el aula la redacción de una situación problemática, el alumno toma como modelo lingüístico lo que se expresa, lo retiene y lo asocia posteriormente con el contenido resolutorio de la situación. El rigor, la precisión y la claridad del lenguaje que se presenta al alumno son de exagerada importancia. Los problemas que se leen a lo largo de la actividad escolar son muchos. $Y$ son muchos los que contienen incorrecciones semánticas, sintácticas y matemáticas en sus enunciados. Se lee, tanto lo que está bien como lo que está mal redactado, y las 
interpretaciones no se sujetan tanto al sentido de su expresión, sino a la intuición de ese sentido. La fijación de ideas claras no puede tener un carácter fragmentario, sino un carácter sistémico, integral. Hay que elevar a una magnitud prioritaria la correcta formulación verbal del problema, poniendo excesivo cuidado en la presentación de su información (p. 42).

Las actividades de evaluación deben formularse para evidenciar que los estudiantes aprenden, comprenden los conceptos y desarrollan sus capacidades y destrezas matemáticas, como demostrar, aplicar, inferir y formular. Problemas enunciados correctamente, con un buen lenguaje literario y matemático, permiten medir, cualitativa y cuantitativamente, que los estudiantes adquieren y desarrollan los contenidos matemáticos plasmados en los libros de texto y que se logran cumplir con los objetivos de aprendizaje.

\section{Métodos y materiales}

La investigación tiene enfoque cuantitativo y los resultados se expresarán en frecuencias y porcentajes. Además, es un estudio descriptivo, por lo que se detallarán los componentes relevantes del desarrollo didáctico de contenidos matemáticos en los libros de texto utilizados por docentes y estudiantes del subnivel Superior de EGB y de los tres años del BGU en Ecuador. Para ello, se realizó una amplia investigación bibliográfica y documental, en la que se analizaron cada uno de los libros de texto, que son el objeto de estudio: tres de la EGB que corresponden a octavo, noveno y décimo años y tres correspondientes al primero, segundo y tercer año del BGU.

Para la elaboración de la guía de observación, presentada en el Cuadro 1, se tomaron en cuenta las sugerencias de Standaert y Troch (2011), sobre: objetivos, contenidos, enfoque, estructura, entre otros, y se agregaron aspectos referentes a evaluación. Asimismo, se agruparon los indicadores en los elementos curriculares (objetivos, contenidos y evaluación), considerados como criterios en esta sección. A través de una escala de tipo Likert, se valoró cada indicador de la siguiente manera: Satisfactorio (S), Medianamente Satisfactorio (MS) y Nada Satisfactorio (NS), y numéricamente con 3, 2 y 1 a cada uno de ellos, respectivamente. El instrumento fue validado por expertos y el coeficiente de confiabilidad, conforme al cálculo del alfa de Cronbach, fue de 0,808 que corresponde a un nivel de confiabilidad alto.

\begin{tabular}{cccc}
\hline Criterios & \multicolumn{2}{c}{ Escala } \\
\hline Objetivos & S & MS & NS \\
\hline
\end{tabular}

1. El objetivo general es presentado de manera clara y precisa.

2. Se formulan objetivos específicos para cada unidad.

3. Los objetivos concuerdan con los contenidos.

4. Los objetivos son dirigidos para todos, incluyendo a las personas con necesidades educativas especiales.

5. Las destrezas con criterio de desempeño (DCD), guardan coherencia con los objetivos.

\footnotetext{
Contenidos

6. Los conceptos son actualizados y poseen base científica sustentada en otros libros.

7. Presenta un resumen preciso al finalizar cada unidad.

8. El desarrollo de los contenidos sigue un proceso lógico y crítico.

9. Los temas son bien explicados y detallados.

10. Contiene ejercicios de repaso que parten de lo simple a lo complejo.
} 
11. Cumple con los requisitos del Currículo Nacional.

12. Contiene temas que responden al interés del estudiante y al contexto.

13. Incorpora las TIC para reforzar y complementar los temas.

14. Los temas tienen secuencia lógica.

15. El texto utiliza vocabulario acorde al nivel de escolaridad y promueve la expresión verbal y escrita.

16. Los ejercicios propuestos tienen relación con los conceptos ya tratados.

17. Los ejemplos presentados guardan coherencia con los conceptos matemáticos.

18. Fomenta el interés y motivación creando vínculos de investigación y trabajo autónomo.

19. Cuenta con variedad de ejercicios para explicar y reforzar los conceptos.

\section{Evaluación}

20. Propone ejercicios o problemas integradores con otras áreas.

21. Las evaluaciones al final de cada unidad tienen relación con los conceptos desarrollados.

22. Los ejercicios y problemas formulados tienen lógica con sus respuestas.

Cuadro 1. Instrumento de observación y análisis de libros de texto por criterios

Para la aplicación de la guía de observación a los libros de texto, se contó con equipos de estudiantes pertenecientes a la Carrera de Pedagogía de las Ciencias Experimentales, Matemática y Física de la Universidad Central del Ecuador, en el período académico 20192019. Se integraron seis equipos, uno por cada texto, quienes fueron capacitados en el uso del instrumento. Finalmente, se utilizó el programa Microsoft Excel para el tratamiento estadístico de la información y la presentación de resultados.

\section{Resultados y discusión}

Los resultados de la investigación y de las observaciones realizadas a los libros de texto analizados de los niveles EGB Superior y BGU, por criterios: objetivos, contenidos y evaluación, y conforme a la escala valorativa: satisfactorio $(S)$, medianamente satisfactorio (MS) y nada satisfactorio (NS), se muestran en cuadros por niveles y años. Esto permite realizar comparaciones entre ellos, contrastando los resultados por criterios con respecto a cada año y nivel.

\subsection{Resultados agrupados por criterios y años del nivel EGB Superior}

En el Cuadro 2 se consideran los criterios: objetivos, contenidos y evaluación, contrastados con los años octavo, noveno y décimo de EGB Superior y sus respectivos porcentajes.

\begin{tabular}{lrcccccccr}
\hline Criterios & \multicolumn{3}{c}{ Objetivos } & \multicolumn{3}{c}{ Contenidos } & \multicolumn{3}{c}{ Evaluación } \\
\hline \multicolumn{1}{c}{ Años } & \multicolumn{1}{c}{ S } & MS & NS & S & MS & NS & S & MS & \multicolumn{1}{c}{ NS } \\
\hline 8vo $^{\text {9no }}$ & 8,00 & 12,00 & 80,00 & 27,14 & 57,14 & 15,71 & 33,33 & 66,67 & 0,00 \\
10 $^{\text {mo }}$ & 5,00 & 40,00 & 55,00 & 27,00 & 53,00 & 20,00 & 50,00 & 50,00 & 0,00 \\
\hline Promedio & 26,67 & 60,00 & 13,33 & 30,95 & 44,05 & 25,00 & 11,11 & 72,22 & 16,67 \\
\hline
\end{tabular}

Cuadro 2. Resultados del análisis de libros de texto por criterios y años del nivel EGB Superior (en porcentajes) 
Es decir, se aprecia que los libros de texto de octavo año tienen el 80,00\% de nada satisfactorio en la formulación de objetivos y solamente el 8,00\% se considera satisfactorio. En lo concerniente a los contenidos, se aprecia que el 57,14\% declara medianamente satisfactorio el desarrollo de estos. Mientras, para la formulación de las actividades de evaluación, se aprecia que el 66,67\% declara medianamente satisfactorio y el 33,33\% la considera satisfactorio.

En el Cuadro 2, para los libros de texto de noveno año se aprecia que el 55,00\% considera nada satisfactorio la formulación de los objetivos y un $40,00 \%$ se observa que es medianamente satisfactorio. Acerca de los contenidos, el 53,00\% de observaciones dicen ser medianamente satisfactorios y, respecto a la evaluación, el 50,00\% para satisfactorio y de igual porcentaje para medianamente satisfactorio.

Finalmente, para el libro de texto de décimo año, en el Cuadro 2 se detallan los siguientes resultados: el 60,00\% para medianamente satisfactorio la formulación de los objetivos, el $44,05 \%$ también medianamente satisfactorio la formulación de los contenidos y, respecto a las actividades de evaluación, el 72,22\% recibe la misma valoración.

En resumen, se puede apreciar que el $49,44 \%$ de observaciones son nada satisfactorias para la formulación de los objetivos expresados en los libros de texto de la EGB Superior; significa que este elemento curricular tan importante en el proceso de enseñanza-aprendizaje es desconocido por los estudiantes. En lo referente a los contenidos, el $51.40 \%$ son desarrollados de forma medianamente satisfactoria y el 63,00\% declara medianamente satisfactorio la formulación de las actividades de evaluación. En este nivel, libros de texto aplicados sin las exigencias y el rigor matemático adecuados producirán aprendizajes poco profundos y con falencias conceptuales que afectarán su posterior desarrollo del pensamiento matemático.

\subsection{Resultados agrupados por criterios y años del nivel BGU}

En cambio, los criterios: objetivos, contenidos y evaluación están contrastados con los años de primero, segundo y tercero de BGU y sus respectivos porcentajes, como se visualiza en el Cuadro 3.

\begin{tabular}{lrcccccccc}
\hline Criterios & \multicolumn{3}{c}{ Objetivos } & \multicolumn{3}{c}{ Contenidos } & \multicolumn{3}{c}{ Evaluación } \\
\hline \multicolumn{1}{c}{ Años } & \multicolumn{1}{c}{ S } & MS & NS & S & MS & NS & S & MS & NS \\
\hline $1^{\text {ro }}$ & 6,66 & 66,66 & 26,66 & 11.91 & 61,90 & 26,19 & 22,22 & 66,67 & 11,11 \\
$2^{\text {do }}$ & 14,28 & 62,86 & 22,86 & 14,29 & 64,28 & 21,43 & 28,57 & 47,62 & 23,81 \\
$3^{\text {ro }}$ & 16,67 & 66,66 & 16,67 & 16,67 & 52,38 & 30,95 & 16,67 & 72,22 & 11,11 \\
\hline Promedio & 12,54 & 65,39 & 22,06 & 14,29 & 59,52 & 26,19 & 22,48 & 62,17 & 15,34 \\
\hline
\end{tabular}

Cuadro 3. Resultados del análisis de libros de texto por criterios y años del BGU (en porcentajes)

En este sentido, se evidencia que los libros de texto del primer año de BGU son medianamente satisfactorios en lo referente a: objetivos $(66,66 \%)$, contenidos $(61,90 \%)$ y evaluación $(66,67 \%)$; mientras que los valores más bajos corresponden a los objetivos con el 6,66\% de satisfacción y a los contenidos con el 11,91\%. La evaluación tiene el 11,11\% de nada satisfactoria. Es recurrente la valoración de medianamente satisfactorios a los objetivos $(62,86 \%)$, los contenidos $(64,28 \%)$ y la evaluación $(47,62 \%)$ en los libros de texto del segundo año de BGU. 
Finalmente, en los libros de texto del tercer año de BGU, el $72,22 \%$ corresponde a medianamente satisfactorios, el criterio evaluación y le siguen los objetivos $(66,66 \%)$ y los contenidos con el 52,38\%. De nada satisfactorios consideran a la evaluación con el 11,11\%.

En resumen, se puede apreciar que los libros de texto del BGU son valorados como medianamente satisfactorios en la formulación de los objetivos, los contenidos y la evaluación con el 65,39\%, el 59,52\% y 62,17\%, respectivamente, y el valor más bajo corresponde al criterio objetivos con el 12,54\%. Estas valoraciones en elementos curriculares importantes resultan insuficientes para que los estudiantes puedan evidenciar conocimientos matemáticos necesarios que permitan continuar con estudios matemáticos más complejos y seguir carreras universitarias importantes, estrechamente ligadas al manejo de la Matemática: ingenierías, economía, arquitectura, estadística, negocios y educativas relacionadas con los números; en lo cual encontrarían muchas dificultades y limitaciones.

\subsection{Resultados agrupados por criterios y niveles EGB Superior y BGU}

Por otro lado, los resultados agrupados por criterios y niveles, comparados entre EGB Superior y BGU son presentados en el Cuadro 4.

\begin{tabular}{|c|c|c|c|c|c|c|c|c|c|}
\hline Criter & \multicolumn{3}{|c|}{ Objetivos } & \multicolumn{3}{|c|}{ Contenidos } & \multicolumn{3}{|c|}{ Evaluación } \\
\hline Niveles & S & $M S$ & $\mathrm{~N}$ & S & $M S$ & NS & $\mathrm{S}$ & MS & NS \\
\hline $\begin{array}{l}\text { EGB } \\
\text { Superior }\end{array}$ & 13,22 & 37,33 & 49,44 & 28,40 & 51,40 & 20 & 31,00 & 63,00 & 0 \\
\hline BGU & 2,54 & 65,39 & 22,06 & 14,29 & 59,52 & 26,19 & 22,48 & 62,17 & 15,34 \\
\hline Promedio & 12,88 & 51,36 & 35,75 & 21,34 & 55,46 & 23,20 & 26,74 & 62,59 & 10,67 \\
\hline
\end{tabular}

Cuadro 4. Resultados del análisis de libros de texto por criterios y niveles EGB Superior y BGU (en porcentajes)

El Cuadro 4 representa los resultados agrupados por los niveles EGB Superior y BGU en cuanto al nivel de satisfacción o concordancia entre los libros de texto y el desarrollo metodológico. Se puede constatar los siguientes promedios: en lo referente al criterio objetivos, el 51,36\% son medianamente satisfactorios, es decir que no son formulados de manera adecuada y no guardan coherencia entre ellos. Sobre los contenidos desarrollados, igualmente, estos son medianamente satisfactorios $(55,46 \%)$, lo que permite señalar que existen falencias y falta de rigor en el abordaje de conceptos matemáticos. Finalmente, en el criterio de evaluación, se tiene un $62,59 \%$ de medianamente satisfactorio, que demuestra que los problemas y ejercicios propuestos son en alguna medida integradores y guardan cierta coherencia lógica con los conceptos matemáticos.

Sin embargo, al contrastar entre los resultados obtenidos en los libros de texto de EGB $(49,44 \%)$ con los del BGU $(65,39 \%)$ se observa que para el elemento curricular -criterioobjetivos, el mayor porcentaje corresponde a los libros de texto del BGU y son medianamente satisfactorios, lo que demuestra que las actividades de enseñanzaaprendizaje están mejor orientadas para la consecución de los logros de aprendizaje. En cuanto al elemento curricular contenidos, existe una pequeña diferencia en los porcentajes tanto de BGU $(59,52 \%)$ como de $\operatorname{EGB}(51,40 \%)$, ambos están en la valoración medianamente satisfactorios. Finalmente, en lo referente al elemento curricular evaluación, los libros de texto de EGB tienen el 63,00\% mientras que para el BGU es del 62,17\% y están en la valoración medianamente satisfactorios. Por lo tanto, mucho depende de los autores y responsables de la publicación de los libros de texto para que haya consistencia entre los niveles EGB y BGU en lo referente a los elementos curriculares: objetivos, contenidos y evaluación. 


\section{Conclusiones}

De los resultados y el análisis descritos sobre los libros de texto utilizados para la enseñanza y aprendizaje de la Matemática, se determinan las siguientes conclusiones:

Los libros utilizados por los estudiantes de la EGB subnivel Superior y del BGU, tienen una estructura que integra en sus unidades temáticas de manera uniforme a: objetivos, contenidos, ejercicios resueltos, ejercicios propuestos, problemas integradores y recomendaciones para las tareas fuera de clase. La investigación y la información analizada muestra que respecto a: objetivos, contenidos y evaluación, estos cumplen medianamente las expectativas de lo que deben ser los libros de texto para la enseñanza de la Matemática en el nivel de EGB Superior y del BGU. Significa, por lo tanto, que los estudiantes que los utilizan no tienen el respaldo metodológico que les permita alcanzar los conocimientos requeridos para desarrollar razonamientos y aprendizajes matemáticos más complejos.

Por lo tanto, si se considera la función que cumplen los objetivos en el proceso de enseñanza-aprendizaje, es preocupante que no estén expresados en algunos casos o que no guarden relación estrecha entre los objetivos generales de las unidades temáticas con los objetivos específicos. Los estudiantes y docentes no tienen el propósito bien definido para aprender y enseñar conceptos esenciales en Matemática. También, se observó que en algunos casos los contenidos no guardan la secuencialidad o secuenciación requerida en su fundamentación y desarrollo teórico, tampoco tienen la profundidad y rigor que estos exigen. Además, preocupa que las actividades de evaluación no estén formuladas para evidenciar la comprensión de conceptos, procesos y desarrollo de capacidades y destrezas que los estudiantes deben demostrar y aplicar. Los ejercicios y problemas matemáticos no son presentados para medir los logros alcanzados ni para integrar los conocimientos desarrollados, o estos problemas son insuficientes.

Finalmente, conviene enfatizar en la pertinencia que debe existir en los libros de texto de los niveles investigados entre los objetivos y la evaluación, debido a que ambos elementos curriculares permiten evidenciar el desempeño docente y de los estudiantes en el proceso de enseñanza-aprendizaje; así también, el rigor con el que se deben desarrollar los contenidos temáticos de tan importante disciplina. Bien se podría recomendar que los libros de texto utilizados para los niveles del EGB Superior y el BGU, necesitan analizarse a profundidad, en los aspectos metodológicos y de desarrollo del proceso de enseñanzaaprendizaje; es decir, en las estrategias y técnicas que motiven a los estudiantes y a los docentes a usarlos de manera óptima.

\section{Agradecimientos}

Reconocemos la participación de los estudiantes de la Carrera de Pedagogía de las Ciencias Experimentales, Matemática y Física de la Universidad Central del Ecuador por su valioso aporte al desarrollo de esta investigación. Asimismo, agradecemos al equipo de investigación "Évariste Galois" de la Universidad Central del Ecuador, de manera especial, a la Lcda. Evelyn Aldaz Guamán, por su apreciable participación en la realización de este trabajo. 


\section{Bibliografía}

Araujo, B. (2009). Planificación y ciclo de aprendizaje. Santillana.

Arribas, J. (2017). La evaluación de los aprendizajes. problemas y soluciones. Revista de Currículum y Formación de Profesorado, 21(4), 381-404. https://www.redalyc.org/pdf/567/56754639020.pdf

Campanario, M. (2001). ¿Qué puede hacer un profesor como tú o un alumno como el tuyo con un libro de texto como éste? Una relación de actividades poco convencionales. Enseñanza de las ciencias: revista de investigación y experiencias didácticas, 19(3), 351-364.

https://www.raco.cat/index.php/Ensenanza/article/view/21753/21588

Choppin, A. (1993). L'histoire des manuels scolaires. Un bilan bibliométrique de la recherche française. Histoire de l'Éducation, 58(1), 165-185. https://www.persee.fr/docAsPDF/hedu_0221-6280_1993_num_58_1_2663.pdf

Constitución de la República del Ecuador. Art. 28. 20 de octubre de 2008 (Ecuador).

Creemers, B. (1991). Effectieve instructie. Een empirische bijdrage aan de verbetering van het onderwijs in de klas. Den Haag, Instituut voor Onderzoek van het Onderwijs.

Duque, R. (1993). La evaluación en la ES Venezolana. Planiuc.

Eyzaguirre B. y Fontaine L. (1997). El futuro en riesgo: nuestros textos escolares. Estudios Públicos, 68(1), 339-354. https://www.cepchile.cl/cep/site/artic/20160303/asocfile/20160303184453/re v68 eyzaguirrefontai.pdf

Fernández, J. (2010). La resolución de problemas matemáticos: creatividad y razonamiento en la mente de los niños. Grupo Mayéutica Educación.

Fernández, M. y Caballero, P. (2017). El libro de texto como objeto de estudio y recurso didáctico para el aprendizaje: fortalezas y debilidades. Revista Electrónica Interuniversitaria de Formación del Profesorado, 20(1), 201-217. https://dialnet.unirioja.es/descarga/articulo/5969918.pdf

Flotts, P., Manzi, J., Barrios, C., Saldaña, V., Mejías, N. y Abarzúa, A. (2016). Aportes para la enseñanza de la matemática. Unesco. https://unesdoc.unesco.org/ark:/48223/pf0000244855

García, A. (2014). El uso del libro de texto de matemáticas en el aula. Universidad de Granada. https://digibug.ugr.es/bitstream/handle/10481/36188/GARCIAMARTINANTONI 0.pdf? sequense $=1$

Ministerio de Educación del Ecuador. (2016). Matemática 3 BGU. Editorial Don Bosco.

Nortes, A. y Nortes, R. (2011). Los libros de texto y la resolución de problemas en la enseñanza-aprendizaje de las matemáticas. Educatio Siglo XXI, 29(2), 67-98. https://revistas.um.es/educatio/article/view/132981/122681

Obaya, A. y Ponce, R. (2007). La secuencia didáctica como herramienta del proceso enseñanza aprendizaje en el área de Químico Biológicas. ContactoS, 63(1), 19-25. http://www2.izt.uam.mx/newpage/contactos/anterior/n63ne/secuencia v2.pdf

Parcerisa, A. (1996). Materiales escolares: cómo elaborarlos, seleccionarlos y utilizarlos. Grao. 
Puente, D. (2016, 18 de julio). Matemática, el dolor de cabeza de los bachilleres de Ecuador. El Comercio. https://www.elcomercio.com/actualidad/matematica-serbachillerecuador-educacion-estudiantes.html

Salcedo, H. (2011). Los objetivos y su importancia para el proceso de enseñanzaaprendizaje. Revista de Pedagogía, 32(91), 113-130. https://www.redalyc.org/pdf/659/65926549007.pdf

Standaert, R. y Troch, F. (2011). Aprender a enseñar. Manthra.

Van Bruggen, J. (1987). Leermiddeleninformatie en leerplanontwikkeling. Notitites van en voor leerplanontwikkelaars, 7(3), 133-141.

Velásquez, M. (2019). El Diferendo por el Territorio Esequibo en los Textos Escolares Venezolanos y Guyaneses [tesis doctoral, Universidad Católica Andrés Bello]. Repositorio Institucional

UCAB. http://biblioteca2.ucab.edu.ve/anexos/biblioteca/marc/texto/AAS9972.pdf

Villardón, M. (2006). Evaluación del aprendizaje para promover el desarrollo de competencias. Educatio Siglo XXI, 24(1), 57-76. https://revistas.um.es/educatio/article/view/153/136 


\section{Autores}

ÁNGEL MONTALUISA-VIVAS obtuvo su título de Magíster en Desarrollo Educativo por la Universidad Central del Ecuador UCE y la Universidad NUR (Bolivia) en 2002. Su título de Posgrado en Informática Aplicada a la Educación lo obtuvo en 1990 en la Universidad Libre de Bruselas (Bélgica). Obtuvo el título de Licenciado en Ciencias de la Educación, Profesor de Enseñanza Media en la Especialización de Matemática y Física por la Facultad de Filosofía, Letras y Ciencias de la Educación de la Universidad Central del Ecuador (1978). Además, realizó un curso de especialización en Metodología de la Matemática en la Universidad de Tsukuba (Japón) en el año 2010. Ha publicado en la revista REIRE de la Universidad de Barcelona, en la Revista ANALES de la Universidad Central del Ecuador y capítulos de libro de la serie APRENDAMOS A EDUCAR. Es integrante del equipo de investigación educativa "Évariste Galois".

Se desempeña como docente titular de la Carrera de Pedagogía de las Ciencias Experimentales, Matemática y Física de la Facultad de Filosofía, Letras y Ciencias de la Educación de la UCE, así como, de tutor de trabajos de titulación en Pregrado y Posgrado en la UCE. Es miembro de la Dirección de Desarrollo Académico de la UCE.

EDGAR SALAS-JARAMILLO obtuvo los títulos de tercer nivel: Licenciado en Ciencias de la Educación, especialización Matemáticas y Física, Facultad de Filosofía, Letras y Ciencias de la Educación de la Universidad Central del Ecuador, año 1970; Ingeniero Civil, opción Estructuras, en la Facultad de Ingeniería, Ciencias Físicas y Matemáticas de la UCE, año 1983; Doctor en Ciencias de la Educación, especialización Matemáticas, en la Facultad de Filosofía de la UCE, año 2000. Obtuvo los títulos de cuarto nivel: Magíster en Educación Superior, Facultad de Filosofía de la UCE, año 2004; Magíster en Educación Matemática por la Universidad Nacional del Chimborazo, año 2007; Doctor en Docencia y Gestión Universitaria (equivalente al PhD Americano) por la Universidad del País Vasco, año 2009.

Se ha desempeñado como docente de las cátedras de Ecuaciones Diferenciales, Física, Laboratorio de Física y Electrónica en la Carrera de Matemáticas y Física (hoy Pedagogía de las Ciencias Experimentales, Matemática y Física) de la Facultad de Filosofía de la UCE, durante más de 45 años. Ha desempeñado las funciones de Jefe del Área de Física, Director de la Carrera y vocal de la Comisión de Evaluación de la Universidad Central. En los últimos 5 años ha publicado artículos en las revistas Cátedra de la Facultad de Filosofía; Anales de la UCE y REIRE de la Universidad de Barcelona. Es miembro del equipo de investigación educativa "Évariste Galois".

LEONARDO CANGA-UNDA Estudiante de la Carrera de Pedagogía de las Ciencias Experimentales, Matemática y Física en la Facultad de Filosofía, Letras y Ciencias de la Educación de la Universidad Central del Ecuador (UCE). Miembro del equipo de investigación educativa "Évariste Galois" de la Facultad de Filosofía, Letras y Ciencias de la Educación de la UCE. Participante en congresos nacionales e internacionales.

ADRIANA PONCE-BENAVIDES Estudiante de la Carrera de Pedagogía de las Ciencias Experimentales, Matemática y Física en la Facultad de Filosofía, Letras y Ciencias de la Educación de la Universidad Central del Ecuador (UCE). Miembro del equipo de investigación educativa "Évariste Galois" de la Facultad de Filosofía, Letras y Ciencias de la Educación de la UCE.

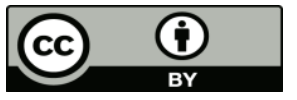

